\title{
Incorporación de raíz de mandioca (Manihot sculenta) en la dieta de cerdos y su efecto sobre variables productivas
}

\author{
Koslowski, H.A.,2, Picot, J.A.'; Sánchez, S. ; Calderón, S. ${ }^{\text {; }}$, Barrientos, F. ${ }^{1}$ \\ ${ }^{1}$ Cátedra de Bioestadística, ${ }^{2}$ Cátedra de Nutrición y Alimentación, Facultad de Ciencias Veterinarias, UNNE, \\ Sargento Cabral 2139, Corrientes (3400), Argentina. E-mail: horaciokoslowski@gmail.com
}

\begin{abstract}
Resumen
Koslowski, H.A.; Picot, J.A.; Sánchez, S.; Calderón, S.; Barrientos, F.: Incorporación de raíz de mandioca (Manihot sculenta) en la dieta de cerdos y su efecto sobre variables productivas. Rev. vet. 28: 2, 121-125, 2017. En un programa de alimentación de cerdos existe una gran variedad de ingredientes que pueden utilizarse en la formulación de la dieta. El objetivo del trabajo fue verificar la conveniencia de utilizar raíz de mandioca en sustitución del maíz como recurso energético en cerdos, mediante la medición de las variables productivas consumo de alimento, ganancia diaria de peso vivo y conversión alimenticia. En el ensayo se utilizó un diseño completamente aleatorizado. Tuvo una duración de 28 días: 7 de adaptación y 21 de medición. La experiencia incluyó 12 animales y cuatro dietas: dieta base (DB) con maíz molido (71\%) más concentrado proteico (29\%); dietas 33\%, 66\% y $100 \%$ : DB a las cuales se les reemplazó el $33 \%$, el $66 \%$ y $100 \%$ del maíz por raíz de mandioca respectivamente. El análisis estadístico se realizó con el software InfoStat/P v.2011. Los resultados indicaron que para las variables consumo de alimento $(1,63-\mathrm{DB} ; 1,56-33 \% ; 1,53-66 \% ; 1,44-100 \%)$ y ganancia diaria de peso vivo $(0,42-\mathrm{DB} ; 0,40-33 \% ; 0,39-66 \% ; 0,36-100 \%)$ no hubo diferencias entre los tratamientos DB, $33 \%$ y $66 \%$, siendo a su vez iguales entre sí los tres niveles de mandioca, mientras que para la conversión (3,91-DB; 3,90-33\%; 3,93-66\%; 4,02-100\%) no hubo diferencias entre los cuatro tratamientos ( $<<0,05)$, lo cual permite concluir que, excepto en el nivel de $100 \%$, la inclusión de la raíz de mandioca en distintos niveles es una opción viable para la alimentación de cerdos.
\end{abstract}

Palabras clave: porcino, alimentos alternativos, energía, parámetros productivos.

\begin{abstract}
Koslowski, H.A.; Picot, J.A.; Sánchez, S.; Calderón, S.; Barrientos, F.: Incorporation of fresh cassava root (Manihot sculenta) in the pig diet and its effect on productive variables. Rev. vet. 28: 2, 121-125, 2017. In a pig feed program there is a wide variety of ingredients that can be used in the formulation of a diet. The objective of this work was to verify the convenience of using cassava root as a substitute for maize as an energy resource in pigs by measuring the variables of food consumption, live weight daily gain and feed conversion. A DCA was used in the assay. It lasted for 28 days, 7 for adaptation and 21 for measurement. The experiment included 12 animals and four diets: base diet (DB) with ground corn (71\%) plus protein concentrate (29\%); diets $33 \%, 66 \%$ and $100 \%$ : DB with replacement of 33,66 and $100 \%$ of maize by cassava root, respectively. Statistical analysis was performed with the software InfoStat $/ \mathrm{P}$ v.2011. From the analysis, for food consumption variables (1.63-DB, $1.56-33 \%, 1.53-66 \%, 1.44-$ $100 \%)$ and daily gain of body weight $(0.42-\mathrm{DB}, 0.40-33 \%, 0.39-66 \%, 0.36-100 \%)$ there were no differences between DB treatments, $33 \%$ and $66 \%$, being the three cassava levels the same, while for the conversion (3,91-DB, 3.90-33\%, 3.93-66\%, 4.02-100\%) there were no differences among the 4 treatments $(\mathrm{p}<0.05)$. This leads to the conclusion that the inclusion of cassava root at different levels, except that for $100 \%$, is a viable option to feed pigs.
\end{abstract}

Key words: porcine, alternative food, energy, productive parameters.

\section{INTRODUCCIÓN}

En los últimos años el mercado mundial de la carne porcina ha experimentado cambios muy importantes, acompañando a los avances tecnológicos llevados

Recibido: 11 noviembre 2016 / Aceptado: 19 abril 2017 a cabo en la producción primaria y las exigencias del consumidor hacia la demanda de productos de calidad, sanos y seguros.

Argentina no ha sido ajena a estos cambios, si bien a un ritmo menor que otros países, como consecuencia de incrementos sucesivos en los precios de los insumos para mezclas balanceadas, manifestando escasa renta- 
bilidad y ausencia de previsibilidad en el negocio, que junto a la caída del poder adquisitivo de la población, se tradujo en una disminución de la producción porcina, llegando en el año 2003 a una faena total de 1.812 .927 cabezas y un consumo de carne porcina de $5,33 \mathrm{~kg}$ de carne habitante/año.

No obstante ello, en los años posteriores se ha observado un constante incremento en la cantidad de cerdos faenados llegándose a registrar 5.986.561 cabezas faenadas en el año 2016, acompañado de un aumento en el consumo de carne porcina expresado en $12,54 \mathrm{~kg}$ de carne por habitante por año ${ }^{14}$.

La producción de carne porcina en zonas con suelos aptos para la agricultura puede convertirse en una alternativa de diversificación especialmente eficaz para pequeños y medianos productores, aumentando sus ingresos y logrando la sustentabilidad de su explotación ${ }^{3}$. Surge entonces que la disminución de la dependencia del empleo de materias primas de origen foráneo reduciría los costos y permitiría a la mayoría de los porcicultores regionales mantenerse en esta actividad productiva a una mayor escala.

Con un sistema alimenticio alternativo donde se incorporen recursos locales en una proporción adecuada, según los requerimientos del animal y el valor nutricional de dichas materias primas, se podría obtener un sistema de producción sostenible desde el punto de vista productivo y económico, sobre todo para aquellos productores de pequeña y mediana escala ${ }^{20}$.

En un programa de alimentación de cerdos existe una gran variedad de ingredientes que se pueden utilizar en la formulación de una dieta. El nivel de inclusión de estos ingredientes en la ración estará determinado por su composición alimentaria, las restricciones nutricionales que tengan para diferentes etapas productivas y el requerimiento de nutrientes que se quiera satisfacer ${ }^{5}$.

En Argentina, el maíz y algunos subproductos industriales representan la mayor fuente de energía para el cerdo, sin embargo, este grano es un elemento básico en la nutrición humana y su baja producción en la región nordeste lo ha convertido en un alimento de importación regional. Esta situación crea la necesidad de buscar otras alternativas que aunque son nutricionalmente inferiores, su utilización a distintos niveles (bajo, medio o alto) puede ayudar a solventar un problema en un momento de crisis y producir adecuados rendimientos productivos ${ }^{1}$.

Existen fuentes alternativas que generalmente presentan ciertas limitaciones nutricionales como ser: deficiencia energética, aminoácidos limitantes y factores antinutricionales. Estas limitaciones pueden afectar el rendimiento productivo, causando una disminución en la eficiencia de conversión de alimentos y un incremento en el costo económico para producir una unidad de producto. Sin embargo, mediante un balance adecuado de nutrimentos y un correcto nivel de utilización, estos productos pueden sustituir adecuadamente las fuentes de energía tradicionales en los alimentos balanceados para cerdos ${ }^{12}$.
La mandioca (Manihot sculenta), podría constituir un recurso sustentable al ser incorporada en la dieta del cerdo. Pertenece a la familia Euphorbiaceae y es una planta de ciclo estival originaria de América. Se desarrolla bien en suelos pobres y ácidos y es tolerante a la sequía, adaptándose a distintos regímenes pluviométricos, desde 600 a $2.000 \mathrm{~mm}$ anuales ${ }^{9}$, condiciones que la hacen ventajosa como una alternativa para la producción animal.

La composición nutricional del tubérculo depende de la variedad, suelo, fertilización y condiciones ambientales. La raíz fresca contiene $65 \%$ de humedad y $35 \%$ de materia seca ${ }^{16}$. Los principales componentes de la materia seca de la harina de mandioca son los carbohidratos, donde un 64 a $72 \%$ corresponden al almidón. Este alto contenido de glúcidos la convierte en una excelente fuente de energía. Para la harina de raíz de mandioca, la mayoría de los investigadores reportan valores de energía metabolizable de alrededor de 3.100 $\mathrm{kcal} / \mathrm{kg}^{4}$.

Las raíces de mandioca contienen factores antinutricionales tales como los cianoglucósidos linamarina y lotaustralina. Al hidrolizarse por acción de la enzima linamarasa ellos liberan el radical $\mathrm{CN}^{-}$que en medio ácido -como el jugo gástrico del animal- generan ácido cianhídrico ${ }^{13}$. En virtud del contenido de dicho ácido, las variedades de mandioca se clasifican en dulces (no tóxicas) cuando el nivel de este ácido no supera los 50 $\mathrm{mg} / \mathrm{kg}$ de producto fresco y en amargas (tóxicas) cuando los valores superan los $50 \mathrm{mg} / \mathrm{kg}^{10}$.

En los animales, los casos agudos de envenenamiento con cianuro terminan en la muerte inmediata, mientras que en los casos menos severos pueden conducir a trastornos gastrointestinales o a disminución del crecimiento. Desde el punto de vista de la nutrición animal la intoxicación con cianuro no es un problema práctico serio cuando se trata del uso de la mandioca. Aún así, se ha evitado el empleo generalizado de las raíces frescas de mandioca ${ }^{8}$.

El objetivo del presente trabajo fue verificar la conveniencia de utilizar raíz de mandioca fresca como sustituto en una alimentación a base de maíz como recurso energético en cerdos en crecimiento.

\section{MATERIAL Y MÉTODOS}

El ensayo se realizó en el módulo de experimentación para cerdos de la Facultad de Ciencias Veterinarias de Corrientes, que posee jaulas individuales, instalación de agua potable (para bebida y limpieza) y depósito de alimento e implementos. En el mismo se evaluó el desempeño de los animales mediante la medición de las variables productivas consumo de alimento, ganancia diaria de peso vivo (GDPV) y conversión alimenticia.

Se utilizaron 12 animales machos castrados en crecimiento, provenientes de cruzas entre las razas Landrace y Yorkshire, de similares características genotípicas (medios hermanos), clínicamente sanos, vacunados 
Tabla 1. Raciones balanceadas con diferentes niveles de sustitución de maíz por mandioca, calculadas en función a la proteína bruta $(18 \%)$, acorde al requerimiento de la categoría de animales.

\begin{tabular}{lcccc}
\hline ingrediente (\%) & DB & $33 \%$ & $66 \%$ & $100 \%$ \\
\hline conc. proteico $(*)$ & 29 & 35,00 & 40,46 & 44,4 \\
maíz (molido) & 71 & 45,07 & 18,41 & 0 \\
mandioca (fresca) & 0 & 19,93 & 41,13 & 55,6 \\
total & 100 & 100 & 100 & 100 \\
\hline
\end{tabular}

DB: dieta base. $33 \%$ : dieta con $33 \%$ de mandioca y $67 \%$ de maíz. $66 \%$ : dieta con $66 \%$ de mandioca y $34 \%$ de maíz. $100 \%$ : dieta con $100 \%$ de mandioca y $0 \%$ de maíz. Los valores 33, 66 y 100 hacen referencia al nivel (\%) de reemplazo de maíz por mandioca, y no a la proporción total en las raciones. $(*)$ : concentrado Santa Sylvina ${ }^{\circledR}$, proteína bruta $38 \%$, extracto etéreo $3 \%$, fibra bruta $7 \%$, cenizas $12 \%$, humedad $11 \%$.

y desparasitados acorde al manejo sanitario habitual de la región. Los animales presentaron un peso inicial de $14 \pm 3 \mathrm{~kg}$.

La mandioca evaluada en el ensayo perteneció al cultivar Pomberí, también conocida como mandioca blanca o pombero. A fin de eliminar la tierra que pudiera estar adherida a la cáscara, las raíces fueron lavadas y luego picadas a un tamaño no superior a $1 \mathrm{~cm}$ mediante una trituradora de forrajes, logrando un producto pastoso de gran contenido de humedad, el cual fue incluido de esta manera en la ración. En la Tabla 1 se detallan las dietas utilizadas en este ensayo: dieta base (DB) con grano de maíz molido como componente energético y tres niveles de sustitución de maíz por raíz de mandioca fresca: 33\%, 66\% y 100\%.

Debido al menor nivel de proteína bruta en la raíz de mandioca con respecto al maíz, fue necesario incrementar el porcentaje de concentrado proteico a medida que aumentaba la proporción de raíz de mandioca en reemplazo del maíz, logrando así mantener las dietas isoproteicas (Tabla 2).

Para las evaluar las variables productivas se efectuó un ensayo donde los cerdos se alojaron en jaulas individuales de 2,75 $\mathrm{m}^{2}$ cada una, siendo alimentados con la dieta base y el recurso alimenticio a probar (tratamientos), calculados según requerimientos de energía y proteína para las categorías de animales a utilizar ${ }^{15}$ (Tablas 1 y 2).

Los cuatro tratamientos fueron asignados aleatoriamente a los animales ( 3 repeticiones) durante 28 días (7 días de adaptación, tanto a las dietas como a la metodología; y 21 días de medición). Se ofrecieron ad libitum las raciones (tratamientos) a evaluar por animal, repartidas en dos comidas diarias, a las 8:00 y 16:00 horas. El alimento rechazado fue recogi-
Tabla 2. Composición química de las dietas expresada en base seca.

\begin{tabular}{lcccc}
\hline ingredientes & DB & $33 \%$ & $66 \%$ & $100 \%$ \\
\hline energía metab.(kcal/kg) & 3270 & 3258 & 3244 & 3235 \\
proteína bruta (\%) & 18,3 & 18,4 & 18,5 & 18,6 \\
extracto etéreo (\%) & 3,3 & 2,7 & 2 & 1,5 \\
fibra bruta (\%) & 3,4 & 4,4 & 5,4 & 6,1 \\
cenizas (\%) & 5,0 & 5,3 & 6,1 & 6,68 \\
extracto no nitrog. (\%) & 70,0 & 69,2 & 68 & 67,1 \\
\hline
\end{tabular}

do y pesado diariamente para determinar el consumo. El agua fue suministrada ad libitum. Con esta prueba se midió el efecto en las variables, consumo de alimento, ganancia de peso vivo, y conversión alimenticia.

Además se realizó un control diario que abarcó los siguientes aspectos: palatabilidad y consumo de alimento ofertado (de visu), evaluación del estado general, vivacidad, apetito, micción, defecación (diarrea/constipación), aspectos del pelaje, piel y mucosas (ictericia/ edema). Para los controles de peso corporal cada animal fue pesado semanalmente con una balanza digital con capacidad de $150 \mathrm{~kg}$.

Con el objeto de efectuar un análisis preliminar de las variables muestrales, se realizó estadística descriptiva, que comprendió: promedio, error estándar y coeficiente de variación. Para el análisis de varianza, la hipótesis nula $\left(\mathrm{H}_{0}\right)$ fue rechazada con un nivel $\alpha$ del $5 \%$.

Para el ensayo se utilizó un diseño completamente aleatorizado (DCA) ${ }^{19}$, cuyo modelo lineal aditivo es: $\mathrm{Y}_{\mathrm{ij}}=\mu+\tau_{\mathrm{i}}+\varepsilon_{\mathrm{ij}}\left(\mathrm{Y}_{\mathrm{ij}}=\right.$ respuesta, $\mu=$ media general, $\tau_{\mathrm{i}}=$ efecto del tratamiento, $\varepsilon_{i j}=$ error aleatorio). Para todos los análisis estadísticos se utilizó el software Infostat/ Profesional (Ver. 2011) bajo entorno Windows.

\section{RESULTADOS Y DISCUSIÓN}

La Tabla 3 muestra las medias aritméticas, errores estándares, coeficientes de variación y resultados del análisis de la varianza de las variables productivas: consumo de alimento, ganancia diaria de peso y conversión alimenticia, analizadas para cada uno de los tratamientos.

Se puede observar que tanto el consumo de alimento como la ganancia diaria de peso vivo no presentaron diferencias significativas entre la dieta base y los dos primeros niveles de sustitución de maíz por raíz

Tabla 3. Estadística descriptiva de las variables muestrales (DB: dieta base, GDPV: ganancia diaria de peso vivo, CV: coeficiente de variación).

\begin{tabular}{lcccccc}
\hline variable & $\mathrm{DB}$ & $33 \%$ & $66 \%$ & $100 \%$ & $\mathrm{CV}$ & $\mathrm{p}$-valor \\
\hline consumo & $1,63 \pm 0,01 \mathrm{a}$ & $1,56 \pm 0,03 \mathrm{ab}$ & $1,53 \pm 0,01 \mathrm{ab}$ & $1,44 \pm 0,07 \mathrm{~b}$ & 5,96 & 0,0608 \\
GDPV & $0,42 \pm 0,01 \mathrm{a}$ & $0,40 \pm 0,02 \mathrm{ab}$ & $0,39 \pm 0,01 \mathrm{ab}$ & $0,36 \pm 0,01 \mathrm{~b}$ & 7,13 & 0,0542 \\
conversión & $3,91 \pm 0,06 \mathrm{a}$ & $3,90 \pm 0,07 \mathrm{a}$ & $3,93 \pm 0,14 \mathrm{a}$ & $4,02 \pm 0,33 \mathrm{a}$ & 7,08 & 0,9659 \\
\hline
\end{tabular}

Medias con una letra común en la misma fila no son significativamente diferentes $(\mathrm{p}<0,05)$. Los valores de las medias están expresados en $\mathrm{kg}$ de materia seca y $\mathrm{kg}$ de peso vivo para consumo y GDPV respectivamente, y $\mathrm{kg}$ de alimento consumido para producir un kg de peso vivo para conversión. 
de mandioca ( $33 \%$ y $66 \%$ ), siendo diferente a la dieta base la dieta con $100 \%$ de raíz de mandioca en reemplazo del maíz. Además fueron iguales entre sí los tres niveles de mandioca. No obstante, de la observación de las medias se puede evidenciar una disminución en los valores de estas variables a media que se incrementa el nivel de raíz de mandioca en las dietas.

Con respecto a la variable conversión alimenticia no se verificaron diferencias significativas entre los cuatro tratamientos, aunque las medias muestran que el tratamiento que incluyó $100 \%$ de raíz de mandioca en reemplazo del maíz fue coincidente con una conversión menos eficiente.

Dicho comportamiento puede explicarse por el incremento en el nivel de fibra bruta a medida que aumenta la proporción de raíz de mandioca en las dietas evaluadas. Según mencionan otros autores ${ }^{7}$, la fibra es un factor que afecta la palatabilidad, digestibilidad y consecuente capacidad de llenado intestinal, lo que a su vez repercute en la ingesta de alimento.

En otros trabajos, utilizando cáscara de mandioca en niveles de $15 \%$ y $30 \%{ }^{18}$ y tres dietas con $10 \%, 35 \%$ y $60 \%$ de afrecho de mandioca ${ }^{17}$ no se observaron diferencias significativas en las variables consumo, ganancia y conversión, adjudicando tal comportamiento a una palatabilidad, volumen de ración y contenido de nutrientes similares en los tratamientos. Esta afirmación podría explicar el menor consumo de materia seca en el tratamiento con mayor nivel de raíz de mandioca fresca observado en nuestro trabajo debido al mayor volumen de materia fresca del mismo.

En otra investigación se observó que cerdos que consumieron dietas con niveles de sustitución de maíz por raíz de mandioca fresca de $16 \%$ y $48 \%$, mostraron mejores ganancias diarias de peso vivo e índices de conversión alimenticia superiores, con respecto a la dieta sin inclusión de mandioca, atribuyendo esto a la alta digestibilidad ileal del almidón de mandioca, lo cual provocaría que los animales satisfagan sus necesidades con menores cantidades de alimento ${ }^{6}$.

Situación parecida fue reportada con niveles de inclusión de $50 \%$ de mandioca y combinaciones de $25 \%$ de mandioca más $25 \%$ de batata (Ipomoea batatas) donde se recomendó incluir mandioca en un $50 \%$ de la dieta, por presentar, si bien algo inferiores, niveles de conversión alimenticia cercanos al alimento testigo ${ }^{2}$.

Asimismo se obtuvieron índices de conversión alimenticia similares al tratamiento sin mandioca, cuando se incorporaron a las dietas niveles de $20 \%$ y $40 \%$ de mandioca ${ }^{11}$, resultados que se aproximan a los del presente trabajo.

En conclusión, los resultados aquí obtenidos sugieren que si bien el máximo nivel de inclusión de raíz de mandioca fresca $(100 \%)$ obtuvo resultados inferiores en las variables analizadas, dicho recurso alimenticio representa una alternativa factible de ser incorporada en la alimentación de cerdos, presentando una buena aceptabilidad por parte de los animales, y cuyo efecto sobre las variables productivas en proporciones de 33\% y $66 \%$ en reemplazo del maíz no difiere significativamente de la dieta base empleada.

\section{REFERENCIAS}

1. Acosta Sosa MA. 2006. Producción de carne porcina y alimentación humana. Memorias $1^{\circ}$ Congreso de producción y carne porcina del NEA, Resistencia, Chaco, Argentina, p. 4-6.

2. Balza O, Labrador J, Alarcón R, Molina G, Cáceres C, Albornoz A. 2011. Uso de la yuca (Manihot esculenta) y la batata (Ipomoea batatas) como alternativa de alimentación nutricional de cerdos. Publ. Unid. Prod. Mérida, Venezuela.

3. Brunori JC. 2013. Producción de cerdos en Argentina: situación, oportunidades, desafios. Public. INTA, Marcos Juárez, Córdoba, Argentina.

4. Buitrago A. 1990. La yuca en la alimentación animal, Publ. Centro Internacional de Agricultura Tropical (CIAT), ISBN 958-9183-10-7. Cali, Colombia.

5. Campabadal CM. 2009. Guía técnica para alimentación de cerdos, Publ.Asociación Americana de Soya, Costa Rica.

6. Domínguez PL, Almaguel R, Añuez M, Ortega A, Ly J. 2012. Yuca fresca y molida en la ceba comercial de cerdos en Cuba. Rev Comp Prod Anim (la Habana) 19: 2-12.

7. Dong GZ, Pluske JR. 2007. The low feed intake in newlyweaned pigs: Problems and possible solutions. Asian-Aust J Anim Sci 20: 440-452.

8. Hill DC. 1973. Chronic cyanide toxicity in domestic animals. In: Chronic cassava toxicity (Nestel B, MacIntyre R, Ed), London, p. 105-111.

9. INTA-Montecarlo. 2008. Producción de mandioca y sus usos. Public. INTA, Misiones, Argentina (inta.gob.ar/documentos/produccion-de-mandioca-y-sus-usos).

10. INTI-Buenos Aires. 2015. Producción, procesamiento, usos y comercialización de la mandioca (www.ue-inti.gob. ar/pdf/publicaciones/cuadernillo22.pdf).

11. Jiménez RF, González C, Ojeda A, Vecchionacce H, Ly J. 2005. Performance traits of finishing pigs fed graded levels of cassava roots and a mixed foliage meal of cassava and trichanthera leaves. Publ Instit Prod Anim, Maracay, Venezuela (www.lrrd.org/lrrd17/2/jime17014.htm).

12. León AM, Angulo I. 1989. Materias primas alternativas para la producción de alimentos concentrados para animales en Venezuela. Rev. Divulg. FONAIAP No 31.

13. Ly J. 1998. Uso de raíces de yuca para cerdos: factores antinutricionales. Rev Comp Prod Porc 5: 3 (La Habana, Cuba).

14. Ministerio de Agricultura, Ganadería y Pesca de Argentina. 2016. Hoja Informativa número 12, diciembre 2016.

15. National Research Council. 1998. Nutrient Requirements of Swine. Subcommittee on Swine Nutrition, Committee on Animal Nutrition, ISBN: 0-309-54988-4, 210 p.

16. Pond WG, Maner JH. 1984. Swine production and nutrition, AVI Publishing Co., Westport, Connecticut, 646 p.

17. Rosales JM, Urbietta H. 1993. Comparativo de niveles de afrecho de yuca en raciones para cerdos en crecimiento 
y engorde, en la zona de Pucallpa. Folia Amazónica, Instituto de Investigaciones de la Amazonía Peruana (IIAP), Iquitos, Perú.

18. Rosales JM, Páucar R. 1996. Uso de la cáscara de yuca en raciones para cerdos en crecimiento. Folia Amazóni$c a$, Instituto de Investigaciones de la Amazonía Peruana (IIAP), Iquitos, Perú.
19. Steel R, Torrie J. 1993. Bioestadística: principios y procedimientos, 2da.Ed., McGraw-Hill, México, 622 p.

20. Tepper R, González C, Figueroa R, Aranque H, Sulbarán L. 2012. Efectos de la alimentación con recursos alternativos sobre la cría de cerdos en cama profunda. Maracay, Venezuela. www.ucol.mx/revaia/portal/pdf/2012/mayo/2.pdf

\section{Revista Veterinaria ingresa a SciELO}

Revista Veterinaria, publicación oficial de la Facultad de Ciencias Veterinarias de la Universidad Nacional del Nordeste (Corrientes, Argentina), ha logrado acceder al Núcleo Básico de Revistas Científicas Argentinas (Nivel 1), luego de calificar adecuadamente en el Centro Argentino de Información Científica y Tecnológica (CAICYT), según Resolución 2485/14 del Consejo Nacional de Investigaciones Científicas y Técnicas (CONICET).

Sobre un puntaje máximo de 33 se obtuvieron 32 puntos. Tal calificación constituye "una garantía de la excelencia de la publicación" (sic) y queda expedita la vía del Portal SciELO (Scientific Electronic Library Online) para los artículos publicados.

En tal calificación gravitó positivamente la circunstancia de haber aumentado el índice de impacto (Scimago-Elsevier) y haber disminuido las autocitaciones. También se tuvieron en cuenta aspectos como la amplia cobertura de la revista, la calidad científica del Comité Editorial, los criterios de evaluación de los artículos, el origen de los autores (locales $60 \%$, nacionales $13 \%$, extranjeros $27 \%$, en idioma inglés), el adecuado balance entre trabajos científicos originales y reseñas bibliográficas (ambos con alta calidad), así como el estricto cumplimiento de la periodicidad semestral y la favorable acogida por indizadores como Cab, J-Gate, Doaj, Ebsco, Gale Cengage, Infocyt, Latindex y Scopus.

Se consolida de esta manera la continuidad de "Revista Veterinaria", que en su acontecer registra más de 50 años de existencia en nuestra Facultad de Ciencias Veterinarias, entidad que en 2017 cumplió el $97^{\circ}$ aniversario de su fundación. 